\title{
A comparative study of magnifying blue laser imaging and magnifying narrow-band imaging system for endoscopic diagnosis of Helicobacter pylori infection
}

\author{
TOMOMITSU TAHARA ${ }^{1}$, KAZUYA TAKAHAMA ${ }^{2}$, NORIYUKI HORIGUCHI ${ }^{1}$, DAI YOSHIDA ${ }^{1}$, \\ TOMOHIKO KAWAMURA ${ }^{1}$, MASAAKI OKUBO ${ }^{1}$, TAKAMITSU ISHIZUKA ${ }^{1}$, MITSUO NAGASAKA ${ }^{1}$, \\ YOSHIHITO NAKAGAWA $^{1}$, TOMOYUKI SHIBATA ${ }^{1}$ and NAOKI OHMIYA ${ }^{1}$ \\ ${ }^{1}$ Department of Gastroenterology, Fujita Health University School of Medicine, \\ Toyoake 470-1192; ${ }^{2}$ Endoscopic Center, Ieda Hospital, Toyota 470-1219, Japan
}

Received July 10, 2017; Accepted July 19, 2017

DOI: $10.3892 /$ br.2017.946

\begin{abstract}
Helicobacter pylori (Hp) infection is a major cause of gastric cancer. The use of proton-pump inhibitors, anti-platelet and anti-coagulant has become widespread in the clinic. Thus, it would be clinically useful to distinguish Hp-positive stomachs by endoscopic findings alone. Blue laser imaging (BLI) is a new image-enhanced endoscopy technique that utilizes a laser light source developed for narrow-band light observation. We investigated the diagnostic ability of magnifying BLI endoscopy to distinguish $\mathrm{Hp}$-positive stomach in cancer free subjects. The data were also compared to the diagnostic ability of magnifying narrow-band imaging (NBI) endoscopy. In total, 215 participants were randomly assigned to the NBI $(n=112)$ and BLI $(n=113)$ groups. The greater curvature of the gastric middle and upper corpus were carefully evaluated with magnifying NBI or BLI. Small, round pits, accompanied with regular honeycomb-like subepithelial capillary networks (SECNs), being regularly interspersed with collecting venules were considered as Hp infection negative, while enlarged or elongated pits with unclear SECNs or dense fine irregular vessels were considered as Hp infection positive. Sensitivity, specificity, positive predictive value (PPV) and negative predictive value (NPV) for the diagnosis of Hp infection for the NBI group was 0.97, 0.81, 0.87 and 0.95, respectively. Sensitivity, specificity, PPV and NPV for the BLI group was 0.98, 0.92, 0.93 and 0.98, respectively. There was no significant difference among the values for the NBI and BLI groups (all P>0.2). In conclusion, the diagnostic ability of magnifying BLI is acceptable, since it is similar to that of magnifying NBI.
\end{abstract}

Correspondence to: Dr Tomomitsu Tahara, Department of Gastroenterology, Fujita Health University School of Medicine, 1-98 Dengakugakubo, Kutsukake-cho, Toyoake 470-1192, Japan E-mail: tomomiccyu@yahoo.co.jp

Key words: Helicobacter pylori infection, magnifying BLI endoscopy, magnifying NBI endoscopy

\section{Introduction}

Helicobacter pylori $(\mathrm{Hp})$ infection is a major cause of gastric cancer (1-3). It has been previously demonstrated that Hp eradication significantly reduces the incidence of metachronous gastric cancer following endoscopic resection of early gastric cancer (4).

Therefore, eradication therapy for patients with Hp-positive chronic gastritis, now covered by the social insurance systems in Japan, is crucial in the assessment of the Hp status when undergoing endoscopic examination (4). Furthermore, the use of proton-pump inhibitors (PPIs), antiplatelet and anti-coagulant has become widespread in the clinic. Consequently, it is imperative to establish diagnostic criteria to distinguish $\mathrm{Hp}$-positive stomachs on the basis of endoscopic findings alone.

Many clinical studies have reported on the diagnostic performance of image-enhanced endoscopy (IEE) techniques such as narrow-band imaging (NBI) for diagnosing endoscopic lesions (5-8). NBI enhances the visualization of the surface vascular pattern using optical filters against a xenon lamp that allows narrow-band light to pass at wavelengths of 415 and $540 \mathrm{~nm}$. Combining the NBI and magnifying endoscopy provides accurate real-time diagnostic performance in gastric neoplastic lesion as well as Hp gastritis compared to conventional white light endoscopy $(9,10)$.

Recently, Fujifilm developed an endoscope system with a semiconductor laser as a light source. The system includes two types of lasers with wavelengths of 410 and $450 \mathrm{~nm}$. The $450 \mathrm{~nm}$ laser irradiates phosphor to produce illumination light similar to that obtained with a xenon lamp. The combination of strong $410 \mathrm{~nm}$ laser light, weak $450 \mathrm{~nm}$ laser light, and fluorescent light enables blue laser imaging (BLI) via narrow-band light observation. Magnifying endoscopy with BLI is useful for evaluating mucosal surface information such as surface blood vessel and structure patterns (11-15). The present study reported that the diagnostic ability of magnifying BLI endoscopy in early gastric cancer is higher than that of conventional white light endoscopy and was similar to that of magnifying NBI endoscopy (15). 

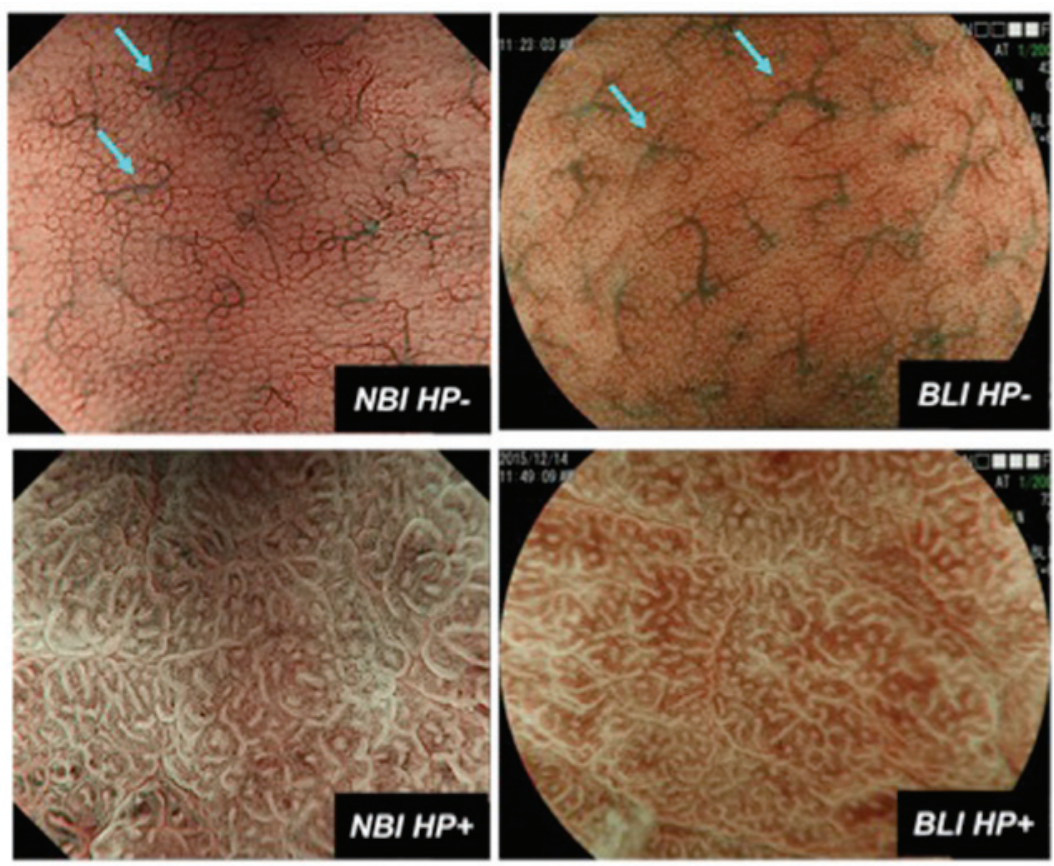

Figure 1. Magnifying NBI (left) and BLI (right) features of Hp infection negative ( $\mathrm{Hp}^{-}$, upper) and positive $\left(\mathrm{Hp}^{+}\right.$, lower) gastric mucosa. Hp ${ }^{-}$gastric mucosa is characterized as small, round pits, accompanied with regular honeycomb-like SECNs, being regularly interspersed with collecting venules (light blue arrow). On the other hand, $\mathrm{Hp}^{+}$gastric mucosa is characterized as enlarged or elongated pits with unclear SECNs or dense fine irregular vessels. NBI, narrow-band imaging; BLI, blue laser imaging; Hp, Helicobacter pylori; SECNs, subepithelial capillary networks.

Magnifying BLI is also potentially useful in distinguishing Hp-positive stomachs as magnifying NBI. We aimed to investigate the diagnostic ability of magnifying BLI endoscopy to distinguish Hp-positive stomach in cancer-free subjects. The data were also compared to the diagnostic ability of magnifying NBI endoscopy.

\section{Patients and methods}

Patients. Study participants were prospectively enrolled from cancer-free patients of the endoscopy Center of Fujita Health University (Toyoake, Japan) and Ieda Hospital (Toyota, Japan) from December 2012 to December 2015. In total, 215 participants were initially invited, and all agreed to participate. All the participants underwent upper gastroscopy for various indications, including annual screening for gastric cancer, secondary complete check-up after barium radiographic examination due to a suspicion of gastric cancer or peptic ulcer disease, and complaints of abdominal discomfort. Of the 215 participants, 112 and 113 participants were assigned to the NBI and BLI groups, respectively, in a random manner. Patients were excluded from the study if they had malignancy, severe systemic disease or advanced chronic liver disease; were receiving non-steroidal anti-inflammatory drugs, PPIs or $\mathrm{H}_{2}$ receptor antagonists; had undergone Hp eradication therapy; and had a history of gastric surgery.

The Fujita Health University School of Medicine approved the protocol (IRB no. HM16-225). Written informed consent was obtained from all participants.

Endoscopic procedure. Prior to endoscopic examination being initiated, 20,000 units of pronase (Pronase MS; Kaken Pharmaceutical Products Inc., Tokyo, Japan) were administered to each participant to remove gastric mucus. The magnifying video endoscope used in the present study was a GIF-H260Z (Olympus Medical Systems Co., Tokyo, Japan) for the NBI group and an EG-L590ZW (Fujifilm Corp., Tokyo, Japan) for the BLI group, respectively.

After the endoscope was inserted, the entire stomach was initially observed with conventional white light to exclude obvious lesions. At least 40 images were captured from the entire stomach, even if there was no obvious lesion.

After the initial survey, the NBI or BLI light source was turned on, and the non-pathological mucosa of the greater curvature of the gastric middle and upper corpus were carefully evaluated with magnification view, the distal tip of the endoscope being attached to the mucosa. During the procedure, a transparent hood, Elastic Touch (Top Corp., Tokyo, Japan), was attached approximately $1.5 \mathrm{~mm}$ distal to the tip of the endoscope to maintain the focal distance. This attachment allowed us to obtaine clear, magnified images more quickly. For the BLI system, the high contrast mode (BLI-contrast) was used to evaluate the gastric mucosal pattern.

To determine Hp status by magnifying NBI or BLI endoscopy, small, round pits, accompanied with regular honeycomb-like subepithelial capillary networks (SECNs), being regularly interspersed with collecting venules were considered to indicate Hp infection-negative gastric mucosa (9,10) (Fig. 1). On the other hand, enlarged or elongated pits with unclear SECNs or dense fine irregular vessels were considered to indicate a Hp infection-positive gastric mucosa (9) (Fig. 1). All of the endoscopic examinations were performed by a single expert endoscopist (TT). Using the endoscopic image of the most predominant magnifying NBI or BLI pattern, mucosal patterns of each case were assessed via consensus among the two expert endoscopists (TT and TS). 

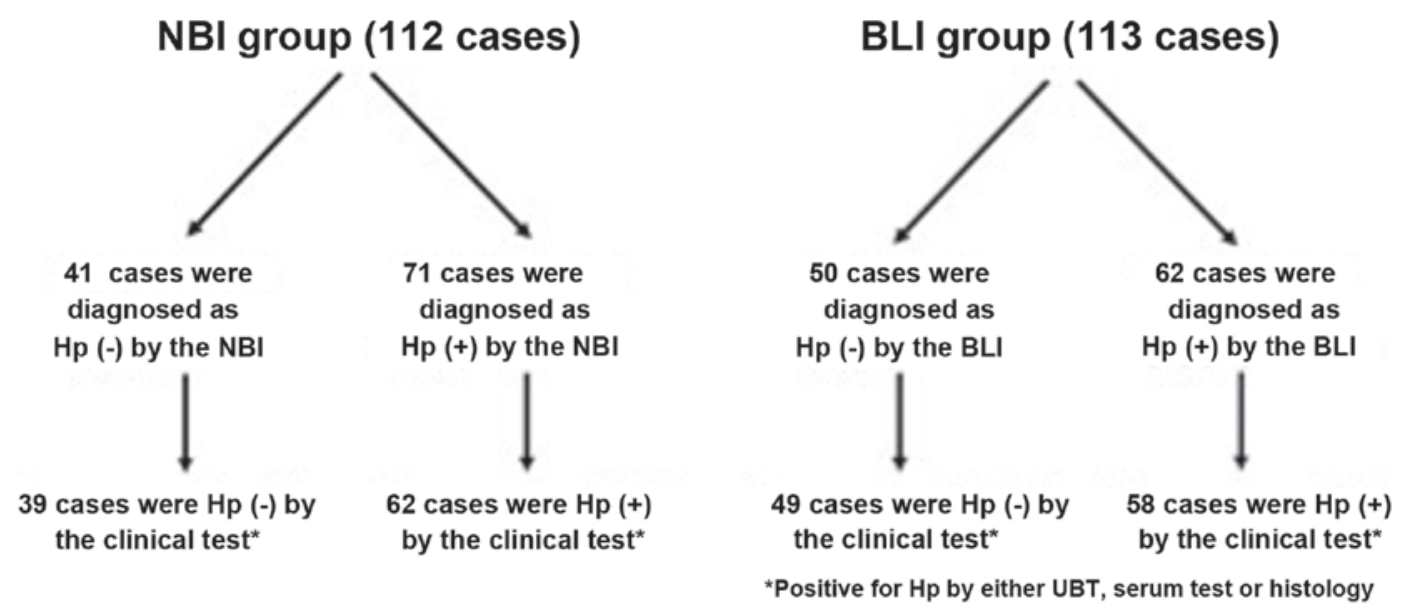

Figure 2. Patient distribution showing the clinical background.

Table I. Clinicopathological characteristics of subjects.

\begin{tabular}{lcc}
\hline Variables & NBI group & BLI group \\
\hline No. of subjects & 112 & 113 \\
Age in years, median (range) & $64(22-87)$ & $63(24-86)$ \\
Sex, male (\%) & $48(42.9)$ & $44(38.9)$ \\
Gastric ulcer, n (\%) & $7(6.3)$ & $2(1.8)$ \\
Duodenal ulcer, n $(\%)$ & $9(8)$ & $4(3.5)$ \\
\hline
\end{tabular}

NBI, narrow-band imaging; BLI, blue laser imaging.

Detection of Hp infection. Urea breath test (UBT), histology using two biopsy specimens from the greater curvature of the gastric antrum and the corpus, as well as the serum titer, were used for the diagnosis of $\mathrm{Hp}$ infection. A positive result in either of these tests was diagnosed as Hp infection. A negative result in any of the tests was diagnosed as Hp infection negative.

Data correction and statistical analysis. The association between endoscopic mucosal patterns using magnifying NBI or BLI endoscopy and Hp infection was evaluated in terms of sensitivity, specificity, positive predictive value (PPV) and negative predictive value (NPV). Sensitivity, specificity, PPV and NPV among NBI and BLI groups were compared using the Fisher's exact test. $\mathrm{P}<0.05$ was considered to indicate a statistically significant difference.

\section{Results}

Clinicopathological characteristics. Clinicopathological characteristics of subjects in the NBI and BLI groups are shown in the Table I. Age and sex were not significantly different between the two groups. During the endoscopy, neoplastic lesions were not detected for either group, while 7 and 9 patients were diagnosed as having gastric and duodenal ulcer in the NBI group. On the other hand, 2 and 4 patients were diagnosed as having gastric and duodenal ulcer in the BLI group, albeit the prevalence of gastric and duodenal
Table II. Sensitivity, specificity, PPV and NPV in magnifying NBI and BLI endoscopy for predicting Helicobacter pylori status.

\begin{tabular}{lccc}
\hline Variables & $\begin{array}{c}\text { Magnifying NBI } \\
\text { endoscopy }\end{array}$ & $\begin{array}{c}\text { Magnifying BLI } \\
\text { endoscopy }\end{array}$ & P-value \\
\hline Sensitivity & 0.97 & 0.98 & 1 \\
Specificity & 0.81 & 0.92 & 0.24 \\
PPV & 0.87 & 0.93 & 0.26 \\
NPV & 0.95 & 0.98 & 0.59 \\
\hline
\end{tabular}

Statistical analysis was performed by the Fisher's exact test PPV, positive predictive value; NPV, negative predictive value; NBI, narrow-band imaging; BLI, blue laser imaging.

ulcers identified in the two groups was not statistically significant $(\mathrm{P}>0.05)$.

Hp infection in the NBI and BLI groups. The mucosal patterns of the greater curvature of the gastric corpus were clearly visualized in all cases in the NBI and BLI groups (Fig. 1). In the NBI groups $(n=112), 41$ cases were considered as Hp infection negative based on the magnifying NBI pattern and 39 cases were diagnosed as $\mathrm{Hp}$ infection negative by the clinical test (UBT, histology and serum titer). On the other hand, 71 cases were considered as $\mathrm{Hp}$ infection positive based on the magnifying NBI pattern and 62 cases were diagnosed as Hp infection positive by the clinical test. As for the BLI group $(n=113)$, 50 cases were considered as Hp infection negative based on the magnifying BLI pattern and 49 cases were diagnosed as Hp infection negative by the clinical test. On the other hand, 62 cases were considered as Hp infection positive based on the magnifying NBI pattern and 58 cases were diagnosed as $\mathrm{Hp}$ infection positive by the clinical test (Fig. 2). Sensitivity, specificity, PPV and NPV for diagnosing Hp infection for the NBI group was $0.97,0.81,0.87$ and 0.95 , respectively. Sensitivity, specificity, PPV and NPV for the BLI group was 0.98, 0.92, 0.93 and 0.98 , respectively. There was no significant difference in values between the NBI and BLI groups (all P>0.2; Table II). 


\section{Discussion}

Results of the present study have shown that the diagnostic ability of magnifying BLI to distinguish Hp-positive stomach seemed to be acceptable for clinical settings, which was similar to the diagnostic ability of magnifying NBI. Magnifying BLI uses narrow-band laser light combined with illumination light. The system provides a high contrast clear view of fine mucosal and capillary structures. Diagnostic effectiveness of BLI endoscopy has been suggested in colorectal tumors and early gastric cancers $(11,15)$. The current study presents new evidence that magnifying BLI endoscopy is useful in distinguishing Hp-positive stomach. Similar to NBI endoscopy, the magnifying BLI feature of Hp-negative gastric mucosa was characterized as small, round pits, accompanied with regular honeycomb-like SECNs, being regularly interspersed with collecting venules, while Hp-positive gastric mucosa was characterized as various sizes of enlarged or elongated pits with unclear SECNs or dense fine irregular vessels. Previous findings have shown that the magnifying NBI patterns of gastric mucosa are closely associated with histological degrees of inflammation and atrophy (9). This is because the histological condition of Hp-positive gastric mucosa is characterized as chronic inflammation with enhanced infiltration of neutrophils and mononuclear cells. Additionally, the continuous destruction and regeneration of new vessels and edema due to severe active inflammation may reflect increased density of irregular microvessels and enlarged or prolonged pits, seen in Hp-positive gastric mucosa. It is possible that magnifying NBI and BLI visualizes fine mucosal patterns and capillary patterns in detail, which suggests the more detailed histological condition of gastric mucosa than the conventional white light endoscopy. Indeed, it has been reported that white light endoscopy itself has limited ability to distinguish Hp-infected subjects (10). It is essential to use IEE such as magnifying NBI and BLI for the endoscopic diagnosis of Hp infection.

Since the diagnostic ability was similar among magnifying NBI and BLI, the magnifying BLI system would serve as a promising tool for the diagnosis of Hp-positive stomach. The use of PPIs, anti-platelet and anti-coagulant has recently become widespread in the clinic. Diagnostic accuracy of a UBT, which is thought to be most sensitive clinical test for detecting Hp, may be decreased by the use of PPIs (16) while the risk of bleeding due to biopsy should not be disregarded for culture of $\mathrm{Hp}$ or histological analysis in patients taking anti-platelet and anti-coagulant. Therefore, the magnifying BLI system would play an important role for patients undergoing such medications. In the present study, we assessed the magnifying NBI and BLI mucosal patterns in the greater curvature of the gastric middle and upper corpus, where the gastric atrophy would be mildest in the stomach. It has also been suggested that magnifying endoscopic assessment of $\mathrm{Hp}$ status should be carried out in mucosa without atrophy (17). The participants in the present study were all cancer-free patients. However, it should be noted that endoscopic assessment of Hp status may become difficult in patients of gastric cancer, especially those that have spread of severe atrophy in the entire stomach.
In Japan, eradication therapy for patients with Hp-positive chronic gastritis is now covered in medical insurance schemes. It has been shown that the magnifying NBI endoscopy can be useful in the assessment of Hp status following eradication therapy $(10,18)$. Our findings may lead to further longitudinal investigation of magnifying BLI endoscopy to determine its clinical utility in such settings.

In conclusion, the present finidngs show that the diagnostic ability of magnifying BLI for predicting Hp status is acceptable, since it is similar to that of magnifying NBI.

\section{References}

1. Parsonnet J, Friedman GD, Vandersteen DP, Chang Y, Vogelman JH, Orentreich N and Sibley RK: Helicobacter pylori infection and the risk of gastric carcinoma. N Engl J Med 325: 1127-1131, 1991.

2. Huang JQ, Sridhar S, Chen Y and Hunt RH: Meta-analysis of the relationship between Helicobacter pylori seropositivity and gastric cancer. Gastroenterology 114: 1169-1179, 1998.

3. Uemura N, Okamoto S, Yamamoto S, Matsumura N, Yamaguchi S, Yamakido M, Taniyama K, Sasaki N and Schlemper RJ: Helicobacter pylori infection and the development of gastric cancer. N Engl J Med 345: 784-789, 2001

4. Fukase K, Kato M, Kikuchi S, Inoue K, Uemura N, Okamoto S, Terao S, Amagai K, Hayashi S and Asaka M; Japan Gast Study Group: Effect of eradication of Helicobacter pylori on incidence of metachronous gastric carcinoma after endoscopic resection of early gastric cancer: An open-label, randomised controlled trial. Lancet 372: 392-397, 2008.

5. Nakayoshi T, Tajiri H, Matsuda K, Kaise M, Ikegami M and Sasaki H: Magnifying endoscopy combined with narrow band imaging system for early gastric cancer: Correlation of vascular pattern with histopathology (including video). Endoscopy 36: 1080-1084, 2004.

6. Ezoe Y, Muto M, Uedo N, Doyama H, Yao K, Oda I, Kaneko K, Kawahara Y, Yokoi C, Sugiura Y, et al: Magnifying narrowband imaging is more accurate than conventional white-light imaging in diagnosis of gastric mucosal cancer. Gastroenterology 141: 2017-2025, 2011.

7. Goda K, Tajiri H, Ikegami M, Urashima M, Nakayoshi T and Kaise M: Usefulness of magnifying endoscopy with narrow band imaging for the detection of specialized intestinal metaplasia in columnar-lined esophagus and Barrett's adenocarcinoma. Gastrointest Endosc 65: 36-46, 2007.

8. Machida H, Sano Y, Hamamoto Y, Muto M, Kozu T, Tajiri H and Yoshida S: Narrow-band imaging in the diagnosis of colorectal mucosal lesions: A pilot study. Endoscopy 36: 1094-1098, 2004.

9. Tahara T, Shibata T, Nakamura M, Yoshioka D, Okubo M, Arisawa $T$ and Hirata I: Gastric mucosal pattern by using magnifying narrow-band imaging endoscopy clearly distinguishes histological and serological severity of chronic gastritis. Gastrointest Endosc 70: 246-253, 2009.

10. Yagi K, Saka A, Nozawa Y and Nakamura A: Prediction of Helicobacter pylori status by conventional endoscopy, narrow-band imaging magnifying endoscopy in stomach after endoscopic resection of gastric cancer. Helicobacter 19: 111-115, 2014.

11. Yoshida N, Hisabe T, Inada Y, Kugai M, Yagi N, Hirai F, Yao K, Matsui T, Iwashita A, Kato M, et al: The ability of a novel blue laser imaging system for the diagnosis of invasion depth of colorectal neoplasms. J Gastroenterol 49: 73-80, 2014.

12. Yoshida N, Yagi N, Inada Y, Kugai M, Okayama T, Kamada K, Katada K, Uchiyama K, Ishikawa T, Handa O, et al: Ability of a novel blue laser imaging system for the diagnosis of colorectal polyps. Dig Endosc 26: 250-258, 2014.

13. Osawa $\mathrm{H}$ and Yamamoto $\mathrm{H}$ : Present and future status of flexible spectral imaging color enhancement and blue laser imaging technology. Dig Endosc 26 (Suppl 1): 105-115, 2014.

14. Miyaki R, Yoshida S, Tanaka S, Kominami Y, Sanomura Y, Matsuo T, Oka S, Raytchev B, Tamaki T, Koide T, et al: A computer system to be used with laser-based endoscopy for quantitative diagnosis of early gastric cancer. J Clin Gastroenterol 49: 108-115, 2015. 
15. Dohi O, Yagi N, Majima A, Horii Y, Kitaichi T, Onozawa Y, Suzuki K, Tomie A, Kimura-Tsuchiya R, Tsuji T, et al: Diagnostic ability of magnifying endoscopy with blue laser imaging for early gastric cancer: A prospective study. Gastric Cancer 20: 297-303, 2017.

16. Shirin H, Frenkel D, Shevah O, Levine A, Bruck R, Moss SF, Niv Y and Avni Y: Effect of proton pump inhibitors on the continuous real time (13)C-urea breath test. Am J Gastroenterol 98 : 46-50, 2003.
17. Yagi K, Nakamura A and Sekine A: Magnifying endoscopy of the gastric body: A comparison of the findings before and after eradication of Helicobacter pylori. Dig Endosc 14: S76-S82, 2002.

18. Okubo M, Tahara T, Shibata T, Nakamura M, Yoshioka D, Maeda Y, Yonemura J, Ishizuka T, Arisawa T and Hirata I: Changes in gastric mucosal patterns seen by magnifying NBI during H. pylori eradication. J Gastroenterol 46: 175-182, 2011. 